\title{
The infant bow shock: a new frontier at a weak activity comet
}

\author{
Herbert Gunell ${ }^{1,2 \star}$, Charlotte Goetz ${ }^{3, \star}$, Cyril Simon Wedlund ${ }^{4}$, Jesper Lindkvist $^{2}$, Maria Hamrin ${ }^{2}$, Hans Nilsson ${ }^{5}$, \\ Kristie Llera ${ }^{6}$, Anders Eriksson ${ }^{7}$, and Mats Holmström ${ }^{5}$ \\ ${ }^{1}$ Royal Belgian Institute for Space Aeronomy (BIRA-IASB), Avenue Circulaire 3, 1180 Brussels, Belgium \\ e-mail: herbert.gunell@physics.org \\ 2 Department of Physics, Umeå University, 90187 Umeå, Sweden \\ 3 Institut für Geophysik und extraterrestrische Physik, TU Braunschweig, Mendelssohnstr. 3, 38106 Braunschweig, Germany \\ e-mail: c.goetz@tu-bs.de \\ 4 Department of Physics, University of Oslo, Box 1048 Blindern, 0316 Oslo, Norway \\ 5 Swedish Institute of Space Physics, Box 812, 98128 Kiruna, Sweden \\ 6 Southwest Research Institute, 6220 Culebra Rd., San Antonio, TX 78238, USA \\ 7 Swedish Institute of Space Physics, Ångström Laboratory, Lägerhyddsvägen 1, 75121 Uppsala, Sweden
}

Received 12 September 2018 / Accepted 15 October 2018

\begin{abstract}
The bow shock is the first boundary the solar wind encounters as it approaches planets or comets. The Rosetta spacecraft was able to observe the formation of a bow shock by following comet 67P/Churyumov-Gerasimenko toward the Sun, through perihelion, and back outward again. The spacecraft crossed the newly formed bow shock several times during two periods a few months before and after perihelion; it observed an increase in magnetic field magnitude and oscillation amplitude, electron and proton heating at the shock, and the diminution of the solar wind further downstream. Rosetta observed a cometary bow shock in its infancy, a stage in its development not previously accessible to in situ measurements at comets and planets.
\end{abstract}

Key words. comets: general - comets: individual: 67P/Churyumov-Gerasimenko - plasmas - shock waves

\section{Introduction}

Shocks are known to form at the interface between plasmas in relative motion: bow shocks upstream of planets and other objects immersed in stellar winds, termination shocks at the edge of the solar system, and shocks in other astrophysical settings (Treumann 2009). Early observations of Earth's bow shock showed a sharp increase in the magnetic field as the solar wind passes the shock, followed by a number of oscillations in the downstream region (Heppner et al. 1967). At comets, bow shocks were observed during the flybys of 21P/GiacobiniZinner (Jones et al. 1986), 1P/Halley (Gringauz et al. 1986a), and 26P/Grigg-Skjellerup (Neubauer et al. 1993). These bow shocks were found to be wider and more gradual than the sharp shocks seen at planets due to solar wind mass loading, and in some instances they have been called "bow waves" instead (Neubauer et al. 1993). These observations were made when the comets were close to perihelion and their bow shocks were fully developed. At comet 1P/Halley additional boundaries were found: a cometopause, which separates the solar wind from the cometary ion environment (Gringauz et al. 1986b), and a diamagnetic cavity (Neubauer et al. 1986).

In the course of its journey, the Rosetta spacecraft (Glassmeier et al. 2007a) followed comet 67P/ChuryumovGerasimenko for two years, providing unprecedented opportunities to observe first, a low-activity state with no identified

$\star$ These authors contributed equally to this work. boundaries (Nilsson et al. 2015), followed by the development of plasma boundaries as a result of the interaction between the comet's atmosphere and the solar wind (Mandt et al. 2016). Between April and December 2015 when the comet was less than 1.8 AU from the Sun, no solar wind ions were observed by Rosetta at its position close to the nucleus, showing that the solar wind was deflected upstream of the spacecraft (Nilsson et al. 2017). This placed the inner coma in a solar wind cavity that has been discussed in terms of a single-particle model (Behar et al. 2017). At about the same time as the solar wind disappeared, the diamagnetic cavity was detected for the first time, and it was then observed frequently until February 2016 (Goetz et al. 2016a,b). Until now, it has been thought that no bow shock was encountered by Rosetta during the mission (Mandt et al. 2016; Simon Wedlund et al. 2017). We present the development of a cometary bow shock from 7 March 2015 when the comet was at a heliocentric distance of $2.2 \mathrm{AU}$ on its way toward perihelion and from the outward journey on 24 February 2016 at 2.4 AU. At these times we can identify an infant bow shock, which we interpret as the structure that will be continuously transformed into a fully developed bow shock at higher comet activity stages. The infant bow shock is asymmetric and it is wider - relative to the distance from shock to nucleus - than fully developed cometary bow shocks. This early stage in bow shock development had never been observed before Rosetta's voyage. Moreover, on 28 March 2015, no diamagnetic cavity was detected in a close flyby, down to a $15 \mathrm{~km}$ cometocentric distance at a $1.99 \mathrm{AU}$ heliocentric distance (Goetz et al. 2016a), and that is closer to the Sun 


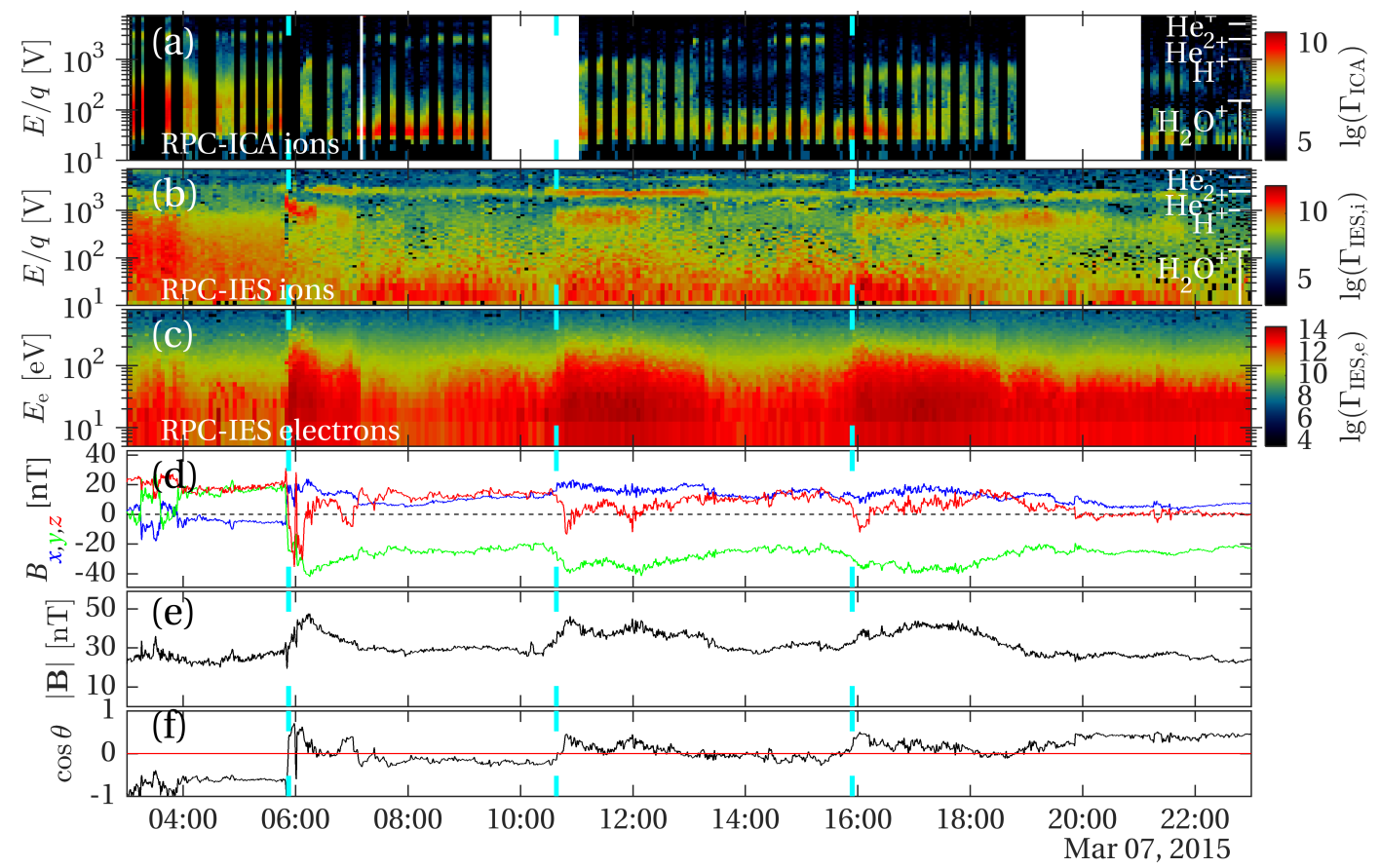

Fig. 1. Rosetta data from 7 March 2015. Panel $a$ : RPC-ICA ion energy spectrum. Panel $b$ : RPC-IES ion energy spectrum. Panel $c$ : RPC-IES electron energy spectrum. The spectra in panels $a-c$ are summed over all viewing directions and the colour-coded quantities $\Gamma_{\mathrm{ICA}}, \Gamma_{\mathrm{IES}, i}$, and $\Gamma_{\mathrm{IES}, e}$ represent the differential particle flux in units of $\mathrm{s}^{-1} \mathrm{sr}^{-1} \mathrm{~m}^{-2} \mathrm{eV}^{-1}$. Panel $d$ : magnetic flux density in CSEQ coordinates measured by RPC-MAG: $B_{x}$ (blue), $B_{y}$ (green), and $B_{z}$ (red). Panel $e$ : magnitude $|\mathbf{B}|$ of the $B$-field. Panel $e$ : estimate of the cosine of the angle between the solar wind electric field vector and the nucleus to spacecraft direction projected onto the $y-z$ plane. Approximate $E / q$ values for $\mathrm{H}^{+}, \mathrm{He}^{2+}, \mathrm{He}^{+}$, and $\mathrm{H}_{2} \mathrm{O}^{+}$ are indicated on the right-hand side of panels $a$ and $b$. The cyan ticks in each panel indicate the times referred to in the text.

than the 2.2 $\mathrm{AU}$ and 2.4 AU heliocentric distance of the infant bow shock observations. Since the cavity expands with decreasing heliocentric distance, this suggests that during the observations presented here no diamagnetic cavity had formed and that the infant bow shock was the only plasma boundary separating the comet and solar wind environments.

\section{Rosetta observations}

Figure 1 shows ion spectra collected by the Ion Composition Analyzer of the Rosetta Plasma Consortium (RPC-ICA; Nilsson et al. 2018), both ion and electron spectra measured by the Ion and Electron Sensor (RPC-IES; Burch et al. 2007), and magnetic flux density from observations by the RPC-MAG instrument (Glassmeier et al. 2007b). During the period on 7 March 2015 shown in Fig. 1, Rosetta was located between 77 and $88 \mathrm{~km}$ from the cometary nucleus, a short distance upstream at $x \approx 50 \mathrm{~km}$ in the body-Centred Solar EQuatorial (CSEQ) coordinate system, where the $x$-axis points toward the Sun, the $z$-axis is the component of the Sun's rotation axes orthogonal to the $x$-axis, and the $y$-axis completes the right-handed frame of reference.

In the ion spectra (Figs. 1a and $\mathrm{b}$ ) four ion species can be distinguished: water group ions at $E / q \lesssim 10^{2} \mathrm{~V}$, protons with $E / q$ values up to about $10^{3} \mathrm{~V}$, alpha particles at $E / q \approx 2.5 \times 10^{3} \mathrm{~V}$, and $\mathrm{He}^{+}$ions around $E / q \approx 5 \times 10^{3} \mathrm{~V}$. These $E / q$ values are shown on the right-hand side of the panels. Three times during the day - near 05:53, 10:38, and 15:54, indicated by cyan ticks in Fig. 1 - the flux of suprathermal $\sim 200 \mathrm{eV}$ electrons suddenly increased and remained high for $1-2 \mathrm{~h}$ (Fig. 1c). This was accompanied by heating of the protons (Figs. 1a and b) and an increase in the magnitude of the magnetic field (Fig. 1e) and in the magnetic field oscillation amplitude at low frequencies, shown for example by the $B_{z}$ curve in Fig. 1d. Low-frequency oscillations in the observed field are expected due to the relative motion of the spacecraft and the inhomogeneous magnetic field associated with the shock. In order to emphasise the low-frequency part of the spectrum the magnetic field has been low-pass filtered with a $-3 \mathrm{~dB}$ cut-off frequency of $10 \mathrm{mHz}$. At the first event (05:53), deceleration of the protons is seen as a decreased $E / q$ value in Fig. 1b. The direction of the magnetic field also changed significantly at this time (Fig. 1d). The RPCICA and RPC-IES instruments are mounted on the spacecraft so that their fields of view complement each other. The solar wind alpha particle beam was present throughout the day, but it shifted between the RPC-ICA and RPC-IES fields of view; for example, at 15:54 the RPC-ICA alpha particle signal decreased, while the corresponding RPC-IES signal increased. A similar shift was seen for the protons at 05:53, when they only appeared in the RPC-IES signal. The $E / q$ value in Fig. $1 \mathrm{~b}$ is seen to decrease and the width of the proton distribution increase in the minutes following that time, showing the deceleration and heating of the proton distribution. A widening of the proton spectrum is also seen for the event starting at 15:54 in Figs. 1a and b. During the rest of the day the proton flux in the two instruments varied in unison indicating a varying proton density.

\section{Observations compared to simulations}

In support of the Rosetta observations, we have used a hybrid plasma model to simulate the interaction between the comet and the solar wind. Cometary $\mathrm{H}_{2} \mathrm{O}^{+}$ions and solar wind $\mathrm{H}^{+}$and $\mathrm{He}^{2+}$ ions are included as particles, and the electrons are treated as a massless charge-neutralising fluid (Holmström et al. 2012; Lindkvist et al. 2018). The simulation run presented in Fig. 2 differs from that of the low ionisation rate case of Lindkvist et al. (2018) in the inclusion of $4.2 \%$ alpha particles among the solar wind ions, where the previously published case had only protons. 

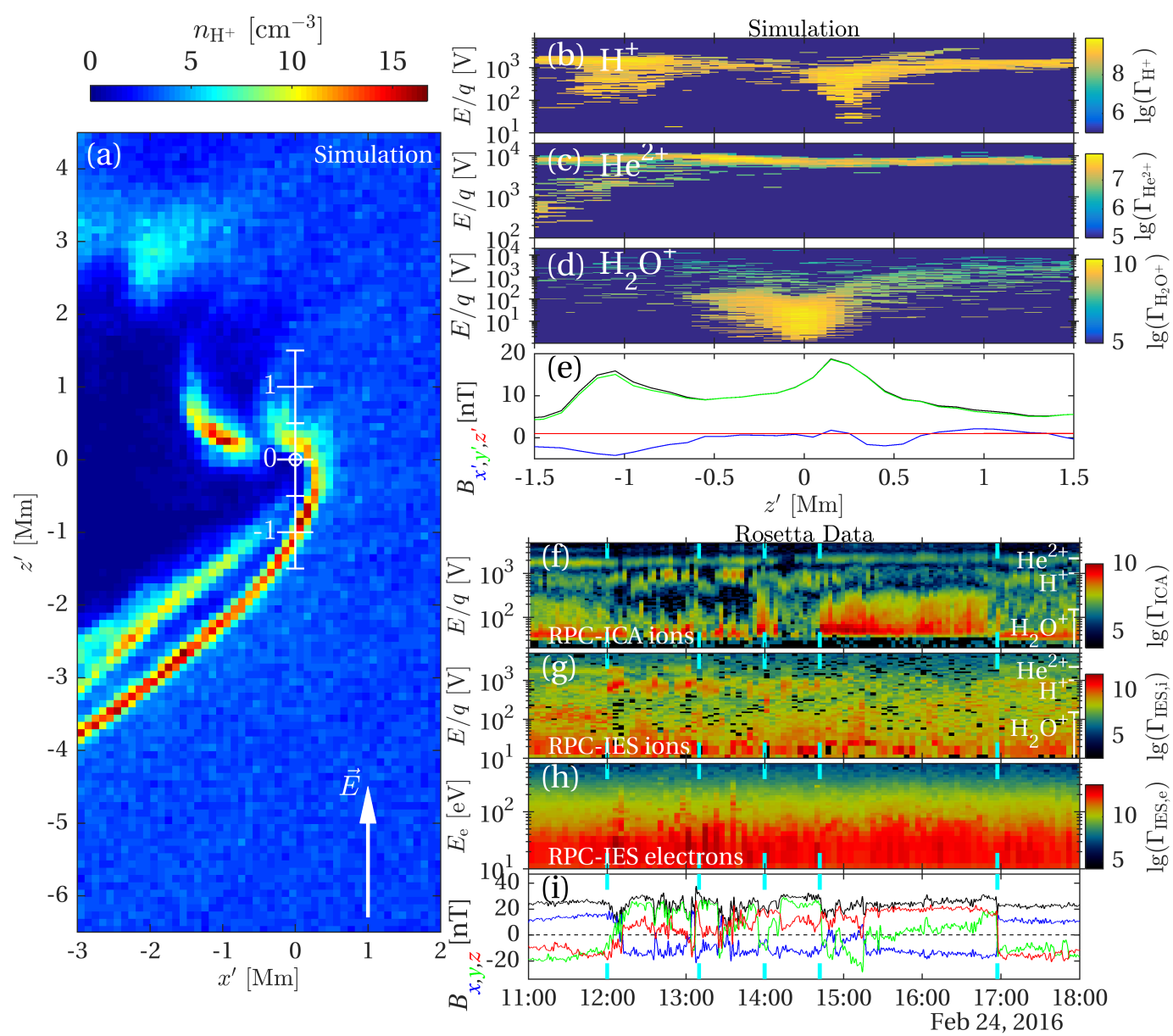

Fig. 2. Simulation and observations on 24 February 2016. Panel $a$ : simulated proton density in the $x^{\prime}-y^{\prime}$ plane. Panel $b$ : simulated proton spectrum along the $z^{\prime}$-axis. Panel $c$ : simulated alpha particle spectrum along the $z^{\prime}$-axis. Panel $d$ : Simulated water ion spectrum along the $z^{\prime}$-axis. Panel $e$ : simulated magnetic field: $B_{x^{\prime}}$ (blue), $B_{y^{\prime}}$ (green), $B_{z^{\prime}}$ (red), and the magnitude $|\mathbf{B}|$ (black). Panel $f$ : RPC-ICA ion energy spectrum. Panel g: RPC-IES ion energy spectrum. Panel $h$ : RPC-IES electron energy spectrum. Panel $i$ : magnetic flux density in CSEQ coordinates measured by RPC-MAG: $B_{x}$ (blue), $B_{y}$ (green), and $B_{z}$ (red), and the magnitude $|\mathbf{B}|$ (black); only the $x$-axis is the same as in the (primed) simulation coordinates. Coordinates $(x, y, z)$ refer to the observations and $\left(x^{\prime}, y^{\prime}, z^{\prime}\right)$ to the simulations. The $x^{\prime}$-axis for panels $b-e$ is indicated in white in panel $(a)$, and the nucleus is located at $\left(x^{\prime}, y^{\prime}, z^{\prime}\right)=(0,0,0)$ and indicated by a white circle in panel a. Approximate $E / q$ values for $\mathrm{H}^{+}, \mathrm{He}^{2+}$, and $\mathrm{H}_{2} \mathrm{O}^{+}$are indicated on the right-hand side of panels $f$ and $g$. The cyan ticks in each panel indicate the times referred to in the text.

All other parameters remain unchanged. The ionisation rate was $1.5 \times 10^{-7} \mathrm{~s}^{-1}$ and the neutral gas production rate $3.2 \times 10^{27} \mathrm{~s}^{-1}$, and the bow shock was not fully developed. The distance between the shock and the nucleus depends on the cometary ion production rate and the solar wind parameters. These parameters change during the observations. Therefore, instead of predicting the exact position of the infant bow shock, we use the simulations to describe the different regions that Rosetta encountered. Due to the slow motion of the spacecraft, the changing solar wind conditions force the infant bow shock to move across an almost stationary spacecraft. We denote the simulation coordinates $x^{\prime}, y^{\prime}$, and $z^{\prime}$, where the $x^{\prime}$-axis points toward the Sun and the $z^{\prime}$-axis is parallel to the solar wind electric field (Fig. 2a).

To aid the comparison between observations and simulations we estimate $\cos \theta$, the $\operatorname{cosine}$ of the angle between the solar wind electric field and the nucleus to spacecraft vector projected onto the CSEQ $y-z$ plane (see Appendix A for a mathematical description). In the absence of an upstream solar wind monitor, the $\cos \theta$ estimate relies on the approximations that $\mathbf{B}$ at the spacecraft has the same direction as $\mathbf{B}$ in the solar wind, and that the solar wind velocity is in the negative $x$ direction. Values of $\cos \theta$ near 1 correspond to spacecraft positions with $z^{\prime}>0$ in
Fig. 2a, and for $\cos \theta$ near -1 the spacecraft is at $z^{\prime}<0$. When $\cos \theta$ is close to 0 the spacecraft is outside the plane of the figure, closer to the $x^{\prime}-y^{\prime}$ plane. Rosetta moves very slowly $\left(\sim 1 \mathrm{~m} \mathrm{~s}^{-1}\right)$ in the reference frame of the comet nucleus, while the infant bow shock moves with respect to that frame. In interpreting the observations it is convenient to describe this relative motion in terms of a spacecraft moving in the rest frame of the infant bow shock, defined by the simulation $\left(x^{\prime}, y^{\prime}, z^{\prime}\right)$ coordinate system. A change in the magnetic field direction will rapidly move it to a different position in the simulation coordinate system. In the 05:53 event on 7 March 2015, $\cos \theta$ changed from negative to positive values, which corresponds to the spacecraft being transported from a position at negative $z^{\prime}$ inside the infant bow shock in Fig. 2a to a position with $z^{\prime}>0$, while $\left|z^{\prime}\right|$ remains approximately the same. This puts Rosetta close to the asymmetrically shaped shock, explaining the appearance of the hot electrons. Spacecraft relative motion with respect to the infant bow shock can also be caused by changes in solar wind pressure and the cometary ion production rate as these also affect the position and shape of the infant bow shock (Lindkvist et al. 2018).

Figures $2 b-d$ show simulated spectra for $\mathrm{H}^{+}, \mathrm{He}^{2+}$, and $\mathrm{H}_{2} \mathrm{O}^{+}$, respectively, along the piece of the $z^{\prime}$-axis indicated 
in white in Fig. 2a. Spectra computed along other lines yield similar results as long as the lines cross the shock. A virtual Rosetta spacecraft flying from high to low $z^{\prime}$ values would first encounter the proton and alpha particle beams of the solar wind. In the $0.1 \mathrm{Mm} \lesssim z^{\prime} \lesssim 0.5 \mathrm{Mm}$ range, the protons are slowed down and heated. The $\mathrm{H}^{+}$population diminishes significantly for $-0.4 \mathrm{Mm} \lesssim z^{\prime} \lesssim 0.1 \mathrm{Mm}$, and for $-0.3 \mathrm{Mm} \lesssim z^{\prime} \lesssim 0.2 \mathrm{Mm}$ a dense water ion distribution appears. Thus, we can identify a region, $0.1 \mathrm{Mm} \lesssim z^{\prime} \lesssim 0.2 \mathrm{Mm}$, where both water and protons are present in significant numbers. Interpreting the data from 7 March 2015 (Fig. 1) as the spacecraft moving in the simulation frame of reference, Rosetta moved between the region with overlapping water ions and proton distributions and that with little water but with a warm proton distribution. Rosetta did not move far upstream enough of the infant bow shock to observe the cold proton beam of the solar wind, although there are instances when a narrow proton distribution indicates that Rosetta was close to that region. During the 05:53 event on 7 March 2015 only, Rosetta moved far enough in the $z^{\prime}$ direction to observe a significant decrease in the water group ion flux. A widening of the alpha particle distribution can also be seen in the simulations for $z^{\prime} \lesssim-0.5 \mathrm{Mm}$, but the spacecraft was not that far away from the nucleus during these observations. Due to their larger gyro-radius the alpha particle scales are longer than the proton scales and therefore the slowing down and heating of alpha particles occurs further downstream and was not observed by Rosetta.

Figures 2f-i show Rosetta data from 24 February 2016. Before 12:00, the presence of warm and slow protons in Fig. $2 \mathrm{f}$ indicates that Rosetta was located downstream of the shock. During the period from 12:00 to 14:00 the infant bow shock moved back and forth over the spacecraft. Several hot electron bursts are seen, accompanied by significant proton energy variations, and magnetic field fluctuations. For example at 13:10 the proton energy dropped from $1 \mathrm{keV}$ to $500 \mathrm{eV}$, suggesting that the spacecraft moved from the upstream to the downstream region. After 14:00 a short interval with both warm protons and water group ions was observed, followed by a period of much lower water ion flux, and from 14:42 to 16:57 a stable period of both warm protons and water ions and a stable magnetic field. This period ended at 16:57 with a directional change of the magnetic field, which moved Rosetta to a different place in the simulation coordinate system. The full 22-24 February 2016 period is shown in Fig. A.1.

Warm ions can at times be observed in the solar wind, away from comets or other solar system objects, as a result of heating at interplanetary shocks (Burlaga et al. 1981; Gosling et al. 1978). However, in the solar wind case, the heating occurs together with an increased bulk speed, which is necessary to maintain the shock in the absence of a planet or an interplanetary body. Since we see a decrease in ion energy, and hence speed, we conclude that what we observe is not a solar wind feature, but the infant bow shock of the comet. Fluxropes have been seen to have temperatures only in the (30-40) eV range (Hu et al. 2004), and instabilities at current sheets can produce similar temperatures (Osman et al. 2012). Thus, neither of these phenomena can be the cause of what we observe in this paper.

\section{Discussion}

Ion dynamics, as described by single-particle models of solar wind protons gyrating in the magnetic field of the coma (Behar et al. 2017), may influence the shape of the infant bow shock. However, our observations of ion and electron heating together with magnetic field enhancements and oscillations at infant bow shocks show that the physics goes beyond a singleparticle description. When the comet was closer to the Sun - with respect to the events reported here - the ion production rate increased, causing the bow shock to move further upstream, and it could not be observed in situ by the Rosetta spacecraft. Observations of cometary water group ions from the upstream regions have indicated the presence of a boundary $4000 \mathrm{~km}$ upstream of the nucleus for a heliocentric distance of 1.4 AU (Nilsson et al. 2007). Whether that was a single frontier or a cometopause had also formed cannot be determined by remote measurements. The infant bow shock we have observed between 2.2 and 2.4 AU constitutes the first formation of what becomes the fully developed bow shock when the comet approaches perihelion. Bow shocks appear around objects that significantly deflect the stellar winds, and the first observation of an infant bow shock probes a regime of their development that previously has been inaccessible to in situ measurements.

Acknowledgements. Work at the Royal Belgian Institute for Space Aeronomy was supported by the Belgian Science Policy Office through the Solar-Terrestrial Centre of Excellence and by PRODEX/ROSETTA/ROSINA PEA 4000107705. Work at Umeå University was funded by the Swedish National Space Board (SNSB project 201/15). Work at the University of Oslo was supported by the Norwegian Research Council grant No. 240000. Data from the Rosetta mission is available through ESA's Planetary Science Archive https://www.cosmos. esa.int/web/psa. The software used in this work was in part developed by the DOE-supported ASC/Alliance Center for Astrophysical Thermonuclear Flashes at the University of Chicago. The hybrid solver is part of the openly available FLASH code and can be downloaded from http://flash.uchicago.edu/. This research was conducted using resources provided by the Swedish National Infrastructure for Computing (SNIC) at the High Performance Computing Center North (HPC2N), Umeå University, Sweden.

\section{References}

Behar, E., Nilsson, H., Alho, M., Goetz, C., \& Tsurutani, B. 2017, MNRAS, 469, S396

Burch, J. L., Goldstein, R., Cravens, T. E., et al. 2007, Space Sci. Rev., 128, 697 Burlaga, L., Sittler, E., Mariani, F., \& Schwenn, R. 1981, J. Geophys. Res., 86, 6673

Glassmeier, K.-H., Boehnhardt, H., Koschny, D., Kührt, E., \& Richter, I. 2007a, Space Sci. Rev., 128, 1

Glassmeier, K.-H., Richter, I., Diedrich, A., et al. 2007b, Space Sci. Rev., 128, 649

Goetz, C., Koenders, C., Hansen, K. C., et al. 2016a, MNRAS, 462, S459

Goetz, C., Koenders, C., Richter, I., et al. 2016b, A\&A, 588, A24

Gosling, J. T., Asbridge, J. R., Bame, S. J., \& Feldman, W. C. 1978, J. Geophys. Res., 83, 1401

Gringauz, K. I., Gombosi, T. I., Remizov, A. P., et al. 1986a, Nature, 321, 282

Gringauz, K. I., Gombosi, T. I., Tátrallyay, M., et al. 1986b, Geophys. Res. Lett., 13,613

Heppner, J. P., Sugiura, M., Skillman, T. L., Ledley, B. G., \& Campbell, M. 1967, J. Geophys. Res., 72, 5417

Holmström, M., Fatemi, S., Futaana, Y., \& Nilsson, H. 2012, Earth, Planets, and Space, 64, 237

Hu, Q., Smith, C. W., Ness, N. F., \& Skoug, R. M. 2004, J. Geophys. Res. (Space Phys.), 109, A03102

Jones, D. E., Smith, E. J., Slavin, J. A., et al. 1986, Geophys. Res. Lett., 13, 243 Lindkvist, J., Hamrin, M., Gunell, H., et al. 2018, A\&A, 616, A81

Mandt, K. E., Eriksson, A., Edberg, N. J. T., et al. 2016, MNRAS, 462, S9

Neubauer, F. M., Glassmeier, K. H., Pohl, M., et al. 1986, Nature, 321, 352 Neubauer, F. M., Marschall, H., Pohl, M., et al. 1993, A\&A, 268, L5

Nilsson, H., Lundin, R., Lundin, K., et al. 2007, Space Sci. Rev., 128, 671

Nilsson, H., Stenberg Wieser, G., Behar, E., et al. 2015, Science, 347, 6220

Nilsson, H., Stenberg Wieser, G., Behar, E., et al. 2017, MNRAS, 469, S252

Nilsson, H., Gunell, H., Karlsson, T., et al. 2018, A\&A, 616, A50

Osman, K. T., Matthaeus, W. H., Hnat, B., \& Chapman, S. C. 2012, Phys. Rev. Lett., 108, 261103

Simon Wedlund, C., Alho, M., Gronoff, G., et al. 2017, A\&A, 604, A73

Treumann, R. A. 2009, A\&ARv, 17, 409 


\section{Appendix A: Estimate of $\cos \theta$}

To aid the comparison between observations and simulations we estimate $\cos \theta$, the $\operatorname{cosine}$ of the angle between the projections onto the $y-z$ plane of the solar wind electric field, $\mathbf{E}_{\mathrm{sw}}=$ $-\mathbf{v}_{\mathrm{sw}} \times \mathbf{B}_{\mathrm{sw}}$, and the nucleus to spacecraft vector,

$\cos \theta=\left(\frac{-\mathbf{v}_{\mathrm{sw}} \times \mathbf{B}}{\left|\mathbf{v}_{\mathrm{sw}} \times \mathbf{B}\right|}\right) \cdot\left(\frac{y \mathbf{u}_{y}+z \mathbf{u}_{z}}{\sqrt{y^{2}+z^{2}}}\right)$,

where $\mathbf{u}_{x}, \mathbf{u}_{y}$, and $\mathbf{u}_{z}$ are unit vectors in the $x, y$, and $z$ directions respectively; $y$ and $z$ are the spacecraft CSEQ coordinates; and $\mathbf{B}$, the field observed at the spacecraft position, is used as an approximation of $\mathbf{B}_{\mathrm{sw}}$. Assuming $\mathbf{v}_{\mathrm{sw}}=-\left|\mathbf{v}_{\mathrm{sw}}\right| \mathbf{u}_{x}$ we obtain

$\cos \theta=\left(\frac{\mathbf{u}_{x} \times \mathbf{B}}{\sqrt{B_{y}^{2}+B_{z}^{2}}}\right) \cdot\left(\frac{y \mathbf{u}_{y}+z \mathbf{u}_{z}}{\sqrt{y^{2}+z^{2}}}\right)$.

The quantity shown in Figs. If and A.1f is $\cos \theta$ computed according to Eq. (A.2).

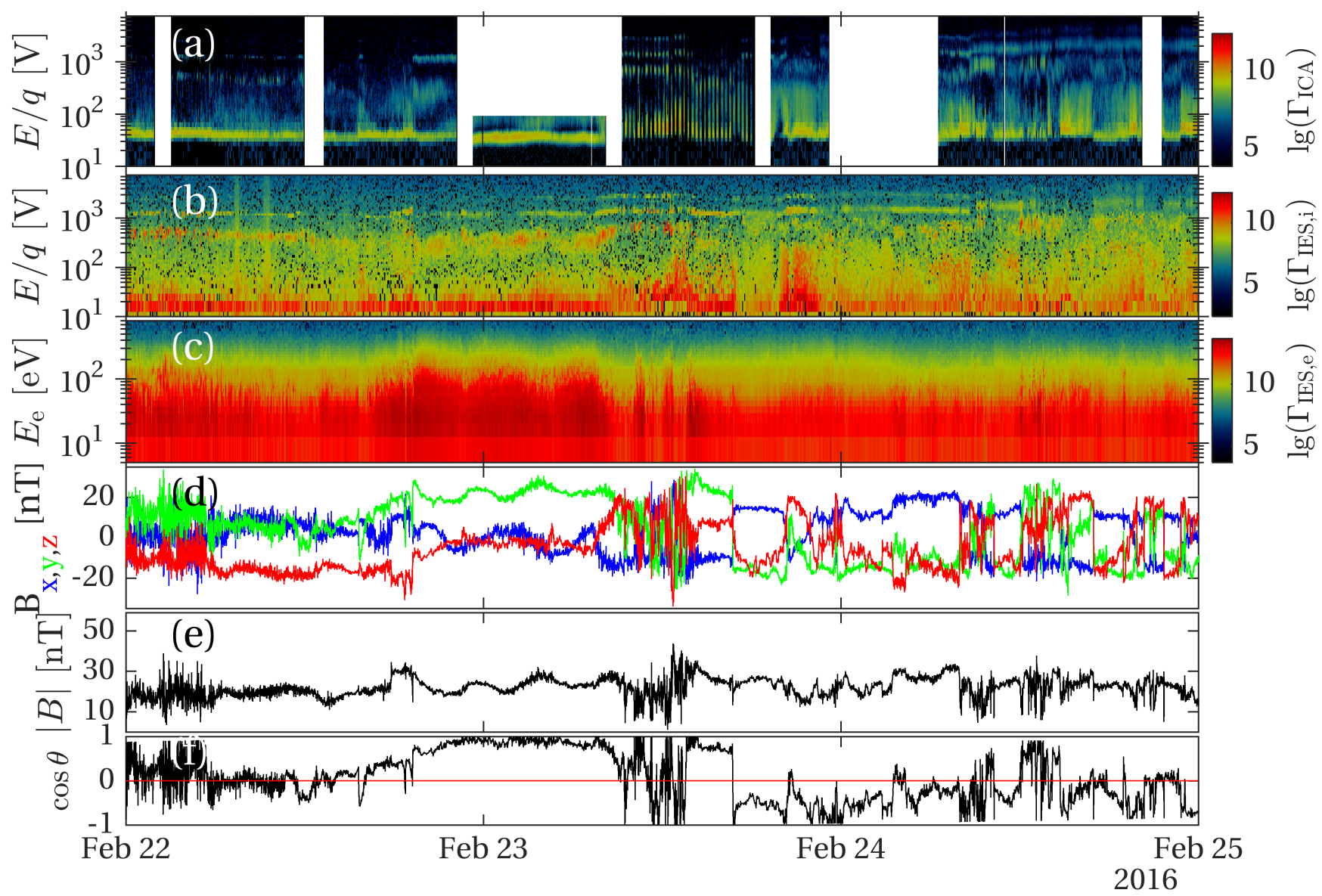

Fig. A.1. Rosetta data from 22-24 February 2016. Panel a: RPC-ICA ion energy spectrum. Panel $b$ : RPC-IES ion energy spectrum. Panel $c$ : RPC-IES electron energy spectrum. The spectra in panels $a-c$ are summed over all viewing directions and the colour-coded quantities $\Gamma_{I C A}, \Gamma_{\text {IES }, i}$, and $\Gamma_{\mathrm{IES}, e}$ represent the differential particle flux in units of $\mathrm{s}^{-1} \mathrm{sr}^{-1} \mathrm{~m}^{-2} \mathrm{eV}^{-1}$. Panel $d$ : magnetic flux density in CSEQ coordinates measured by RPC-MAG: $B_{x}$ (blue), $B_{y}$ (green), and $B_{z}$ (red). Panel $e$ : the magnitude $|\mathbf{B}|$ of the $B$-field. Panel $f$ : estimate of the cosine of the angle between the nucleus to spacecraft direction and the electric field vector, defined by Eq. (A.2). 